\title{
miR-597-3p inhibits invasion and migration of thyroid carcinoma SW579 cells by targeting RAB23
}

\author{
Hu-Ling Wen ${ }^{1}$, Zheng-Min Xu' ${ }^{2}$, Shi-Yu Lin ${ }^{1}$, Dan Wen ${ }^{1}$, Jian-Ping Xie ${ }^{3}$ \\ ${ }^{1}$ Department of Nuclear Medicine, Affiliated Hospital of North Sichuan Medical College, Nanchong, Sichuan, China \\ 'Institute of Medicine, North Sichuan Medical College, Nanchong, Sichuan, China \\ ${ }^{3}$ Nuclear Medicine, North Sichuan Medical College, Nanchong, Sichuan, China
}

\begin{abstract}
Introduction: Nowadays, it is indicated that miRNA was anomaly expressed in tumour. Previous studies have shown that miRNAs can regulate the proliferation, invasion, and migration of cancer cell-related processes. Meanwhile, current investigations show that RAB23 also plays an important role in cancer cell-related processes. But the potential mechanism remains unclear.

Material and methods: SW579 cells were selected and transfected with miR-597-3p mimics. Then the expression of miR-597-3p and RAB23 were measured by quantitative real-time polymerase chain reaction (PCR) and western blotting, respectively. Subsequently, the abilities of proliferation, invasion, and migration of SW579 cells were researched. For further study, the Luciferase reporter assay proved that miR-597-3p could target the expression of RAB23, and the proteins of invasion and migration were also measured to clear the mechanism. Results: After being transfected with miR-597-3p mimics, the expression of miR-597-3p was remarkably increased and RAB23 was significantly decreased. The abilities of proliferation, invasion, and migration also decreased significantly. The miRTarase Database predicated and Luciferase reporter assay proved that RAB23 was the target gene of miR-597-3p. The expression of matrix metalloproteinase (MMP)-2, MMP-9, and N-cadherin was down-regulated, and the expression of E-cadherin was up-regulated.

Conclusion: miR-597-3p could reduce the proliferation, invasion and migration abilities of SW579 cells, which may be related to the targeted inhibition of RAB23 expression and down-regulation of the expression levels of MMP-2, MMP-9 and N-cadherin proteins of SW579 cells. (Endokrynol Pol 2021; 72 (1): 22-28)
\end{abstract}

Key words: miR-597-3p; invasion; migration; thyroid carcinoma; SW579 cells

\section{Introduction}

Thyroid carcinoma (TC) is an aggressive malignancy, which originates from parafollicular C cells or follicular thyroid $[1,2]$. In a recent study, the global mortality rates and incremental incidence of TC in patients were shown to have increased significantly; in addition, about $20 \%$ of TC patients develop regional or local recurrences and about $15 \%$ develop distant metastases. Also, despite traditional surgery such as radioiodine therapy or surgical for TC patients, in most cases it is an incurable disease $[3,4]$. Thyroid squamous cell carcinoma is a relatively rare malignancy, constituting $<1 \%$ of all TCs, which has a low incidence, high degree of malignancy, and poor prognosis [5]. More importantly, the abilities of cell proliferation, migration and invasion are the key factors leading to poor prognosis and low survival rate of thyroid squamous cell carcinoma. MicroRNAs (miRNAs), a class of noncoding and small RNAs, play a critical role in gene expression by targeting binding of the 3'-untranslated region, and thus could regulate cellular biological processes such as cell growth, cycle, and apoptosis [6, 7]. Recently, more and more miRNAs have been found to be involved in the development of tumours and effected the metastasis of tumours [8]. In thyroid carcinoma, researchers found that the expression of miR-577 was significantly down-regulated in TC tissue and TC-1 cell lines. Up-regulating the expression of miR-577 and targeting the supression of SphK2 could inhibit the proliferation, invasion, and migration abilities of TC-1 cell lines in vitro [9]. In addition, miR-582-5p significantly down-regulated in the salivary adenoid cystic carcinoma and significantly influenced the proliferation, invasion, and migration abilities of salivary adenoid cystic carcinoma cells by targeting FOXC1 [10]. RAB23, a class of Ras-related small GTPase family, was first extracted from the brain, and subsequently the role of RAB23 was reported in multiple types of tumour and was demonstrated to be involved in the development and occurrence of lung cancer, liver cancer, gastric cancer, and breast cancer, as well as being closely related to cancer progression [11,12]. The study showed that miR-338-3p bound directly to the $3^{\prime}$-untranslated region of RAB23 and was negatively correlated with 
RAB23 expression, and then suppressed prostate cancer growth in vivo, as well as cell proliferation, invasion, and migration [13]. Other reports also proved that RAB23 correlated with cancer progression; in ovarian cancer, the high expression of RAB23 was associated with a reduced disease-free survival time and overall survival. Subsequently, RAB23 was a target to inhibit drug resistance and improve the prognosis of patients with ovarian cancer [14]. However, as far as we know, the role of miR-597-3p in thyroid carcinoma and its relationship with RAB23 has not yet been reported. In the present study, the expression of miR-597-3p was up-regulated, and then the abilities of proliferation, invasion, and migration and the expression of RAB23 were measured in SW579 cells, which were derived from squamous carcinoma.

\section{Material and methods}

\begin{abstract}
Material
Dulbecco's modified Eagle's medium (DMEM) and foetal bovine serum (FBS) were purchased from Solarbio (Bei Jing, china). MiR597-3p mimics and miR-597-3p NC were provided by Ribobio Biotechnology Co. (Guangzhou, China). Matrix metalloproteinase (MMP)-2, MMP-9, N-cadherin, E-cadherin, $\beta$-actin, and IgG-HRP antibody were purchased from Solarbio Biotechnology Co. (Bei Jing, China). RAB23 antibody was purchased from Heng Fei Biotechnology Co. (Shang Hai, China). ProFlex ${ }^{\mathrm{TM}}$ polymerase chain reaction (PCR) system was manufactured by Thermo Fisher company in USA. GelDoc XR+ Gel imager was manufactured by BIO-RAD company in USA.
\end{abstract}

\section{Cell culture}

The human thyroid cancer cell line (SW579) was purchased from the Shanghai Institutes for Biological Sciences, Chinese Academy of Sciences (Shanghai, China). The cells were cultured in DMEM, which was supplemented with $10 \%$ FBS, $100 \mu \mathrm{M}$ streptomycin, and $100 \mu \mathrm{M}$ penicillin and placed in an incubator at $37^{\circ} \mathrm{C}, 5 \% \mathrm{CO}_{2}$ and saturated humidity. The DMEM medium was updated every two days, and the cells were passaged by trypsin digestion when the cell density reached $90 \%$ confluence.

\section{Cell transfection}

The SW579 cells were collected and suspended by FBS-free DMEM medium. $1 \times 10^{5}$ cells $/ \mathrm{mL}$ were seeded in 24 -well plates and cultured overnight. The SW579 cells were divided into three groups: (1) miR-597-3p mimic group (transfected with a miR-597-3p mimic sequence); (2) miR-597-3p NC group (transfected with a scrambled sequence); and (3) control group (no sequence transfected). The cells were transfected by Lipofectamine 2000 for $5 \mathrm{~h}$, and the DMEM medium was updated after transfecting.

\section{Quantitative real-time PCR}

The total RNA of SW579 cells were isolated by TRIzol reagent. cDNA was synthesised by a PrimeScript Reverse Transcriptase kit. The relative expression of miR-597-3p was analysed by quantitative real-time PCR apparatus. Reaction system: $0.5 \mu \mathrm{L}$ forward and reverse sequence, $2 \times \operatorname{Mix} 10 \mu \mathrm{L}, 1 \mu \mathrm{L}$ cDNA template, and $8 \mu \mathrm{L} \mathrm{H}_{2} \mathrm{O}$. Reaction conditions: $95^{\circ} \mathrm{C} 10 \mathrm{~min}$, followed by 30 cycles $\left(94^{\circ} \mathrm{C} 30 \mathrm{~s}\right.$, $60^{\circ} \mathrm{C} 1 \mathrm{~min}, 78^{\circ} \mathrm{C} 30 \mathrm{~s}$ ), and $72^{\circ} \mathrm{C} 5 \mathrm{~min}$. The relative expression of miR-597-3p was calculated by $2^{-\Delta \Delta C t}$ method. The sequence of miR597-3p and U6 was as follows:
- miR-597-3p:

- F: 5'-ACACTCCAGGTCGGGTGGT-3'

- R: 5'-ATTGGAACGATACAGAGAAGATT-3' - U6:

- F: 5'-TGGTGTCGTGGAGTCG-3'

- R: 5'-GGAACGCTTCACGAATTTG-3'.

\section{Western blotting assay}

The SW579 cells with transfected were lysed by RIPA buffer, then the total protein was extracted from the cell lysates and the concentration was quantified by BCA protein analysis kit. The target proteins were separated by $10 \%$ sodium dodecyl sulphatepolyacrylamide gel electrophoresis (SDS-PAGE), and proteins were transferred onto polyvinylidene fluoride (PVDF) membranes. After incubating for $1 \mathrm{~h}$, they were blocked by $5 \%$ skimmed milk. The monoclonal rabbit-anti-human primary antibodies (dilution 1:100) and $\beta$-actin as an internal reference were added, and incubated at $4^{\circ} \mathrm{C}$ for $24 \mathrm{~h}$. Next, the membranes were incubated with goat anti-rabbit horseradish peroxidase (HRP)-conjugated secondary antibody at $25^{\circ} \mathrm{C}$ for $2 \mathrm{~h}$. Finally, the greyscale images of protein were analysed with Gel-Pro Analyzer 4.0.

\section{Cell counting kit 8 (CCK-8) assay to evaluate the cell viabilities}

The transfected SW579 cells were suspended by FBS-free DMEM medium and seeded into 96-well plates at a density of $8 \times 10^{3}$ cells/ /well. The DMEM medium was abandoned and $180 \mu \mathrm{L}$ DMEM, $20 \mu \mathrm{L}$ CCK-8 was added to each well after culturing for $24 \mathrm{~h}$. The supernatant was removed after $2 \mathrm{~h}$, and then the absorbance value at $450 \mathrm{~nm}$ wavelength was measured by microplate reader; meanwhile, $10 \%$ of CCK- 8 were regarded as blanks. All experiments were repeated three times and measured three times; the final results were obtained by calculating the average of all results.

\section{Cell invasion and migration assays}

The invasion abilities of SW579 cells were estimated by Transwell chamber assay. SW579 cells were seeded into the upper chamber, which was covered with $50 \mu \mathrm{L}$ Matrigel Basement Membrane Matric $(2 \mathrm{mg} / \mathrm{mL})$ and $200 \mu \mathrm{L}$ FBS-free DMEM medium was added. Meanwhile, the $600 \mu \mathrm{L}$ DMEM with $10 \%$ FBS was added into the lower chamber. Subsequently, after incubation at $37^{\circ} \mathrm{C}$ for $24 \mathrm{~h}$, the supernatant was abandoned and the SW579 cells remaining in the upper chamber were removed using cotton swabs. Invaded cells were fixed with methanol for $15 \mathrm{~min}$ and subsequently stained with $0.1 \%$ crystal violet. The migration abilities of SW579 cells were estimated by following the aforementioned method, except for the lack of Matrigel basement membrane matric. All experiments were carried out in triplicate.

\section{Luciferase reporter assay}

The potential target genes of miR-335-5p were predicted by the miRTarase Database. In order to confirm the sequence of RAB23 3'-UTR containing the miR-597-3p binding region, the sequence was cloned into pGL3 Luciferase reporter vector. The potential miR-597-3p binding sites were mutated. The wild type (wt) RAB23 3'-UTR vector or mutant (mt) RAB23 3'-UTR vector and miR-597-3p mimics were co-transfected into SW579 cells by using the Lipofectamine ${ }^{\mathrm{TM}}$ 2000 method. Luciferase activity was detected by dual-luciferase reporter assay kit.

\section{Statistical analysis}

All data were assessed by SPSS software 22.0 (Chicago, USA) and were expressed as means \pm standard deviation (SD). Paired $t$ test was used for within group and one-way ANOVA for between group comparisons; $\mathrm{p}<0.05$ was considered to indicate a statistically significant difference. 


\section{Results}

The expression of miR-597-3p and RAB23 in SW579 cells

In order to detect the role of miR-597-3p in SW579 cell invasion and migration, SW579 cells were transfected with miR-597-3p mimics. The level of miR-597-3p with miR-597-3p mimics group was significantly increased compare with the control group and miR-597-3p NC group by 1.45 fold and 1.44 fold (Fig. 1A). To analyse the changing of RAB23 after transfection, the expression of RAB23 was detected, the RAB23 level was decreased compared with the control group and miR-597-3p NC group by nearly 0.48 fold and 0.49 fold, respectively $(p<0.05$, Fig. $1 B$ and $1 C)$.

\section{The cell viabilities were decreased in SW579 cells after transfection}

To evaluate the effect of miR-597-3p on SW579 cell viabilities after transfection with miR-597-3p mimics, CCK-8 assay was implemented. From Figure 2A, the cell viability of the miR-597-3p mimic group was $60.26 \%$, which was lower than the miR-597-3p NC group $(92.12 \%)$ and the control group $(90.28 \%)$ $(\mathrm{p}<0.05)$.

\section{The cell invasion and migration abilities} were decreased in SW579 cells after transfection Transwell chamber assay showed that, compared with control group and miR-597-3p NC group, the miR-597-3p mimic group (miR-597-3p overexpression) significantly decreased the invasion and migration abilities of SW579 cells ( $p<0.05$, Fig. 2B and 2C). Furthermore, increasing the level of miR-597-3p significantly inhibited the invasion and migration abilities of SW579 cells.

\section{The levels of protein invasion and migration were decreased in SW579 cells after transfection} To further study of the invasion and migration abilities of SW579 cells after transfection with miR-597-3p mimic sequences, the proteins of invasion and migration were detected. Compared with the control group and miR-597-3p NC group, the miR-597-3p mimic group had remarkably decreased levels of MMP-2, MMP-9, and $\mathrm{N}$-cadherin $(\mathrm{p}<0.05)$, and significantly increased levels of E-cadherin ( $p<0.05$, Fig. 3B and $3 \mathrm{C})$.

\section{RAB23 target of miR-597-3p}

To prove RAB23 was the target gene of miR-597-3p, the miRTarase Database and Luciferase reporter assay were

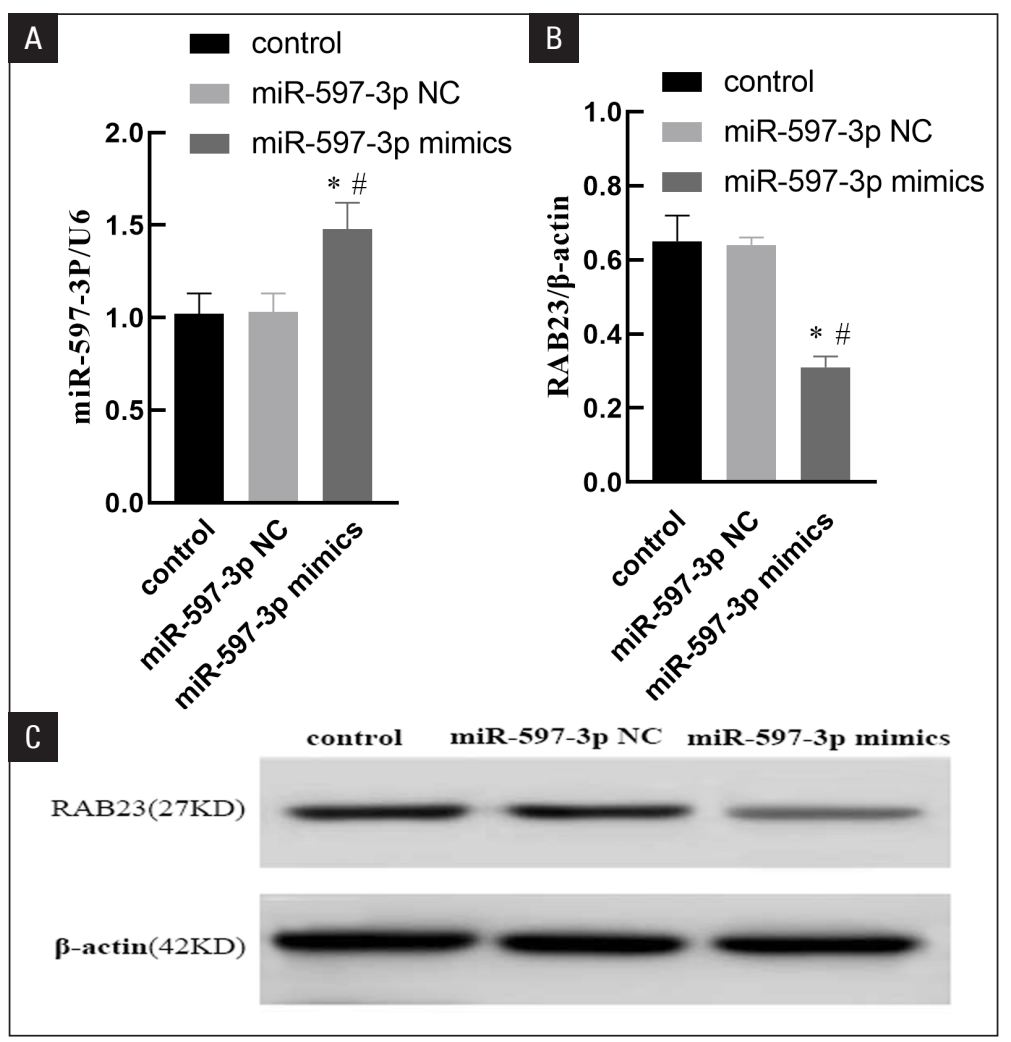

Figure 1. The expression of miR-597-3p (A) and RAB23 (B and C) in SW579 cells. Data are expressed as mean $\pm S D(n=3)$. ${ }^{*} p<0.05$ vs. control group; ${ }^{*} p<0.05$ vs. miR-597-3p NC group 


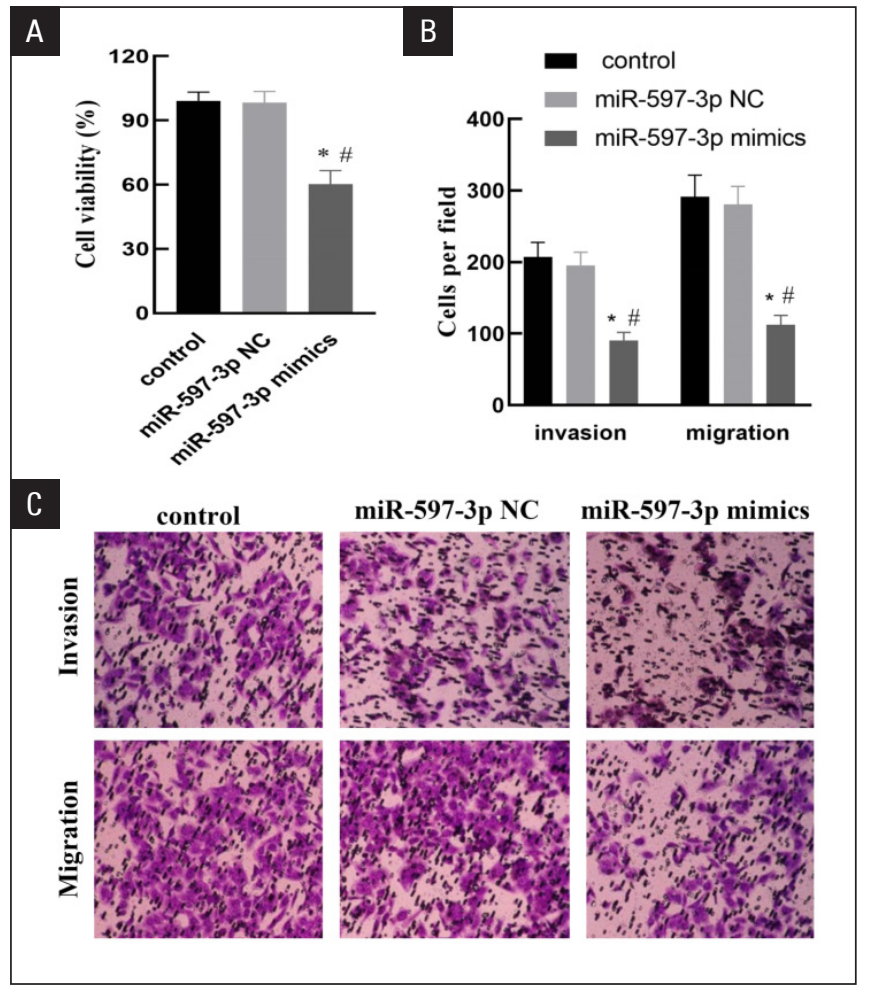

Figure 2. A. The cell viabilities of SW579 cells after transfection. Data are expressed as mean $\pm S D(n=3)$. B, C. The invasion and migration abilities of SW579 cells after transfection. Data are expressed as mean $\pm S D(n=3)(\times 200) .{ }^{*} p<0.05$ vs. control group; ${ }^{\#} p<0.05$ vs. miR-597-3p NC group

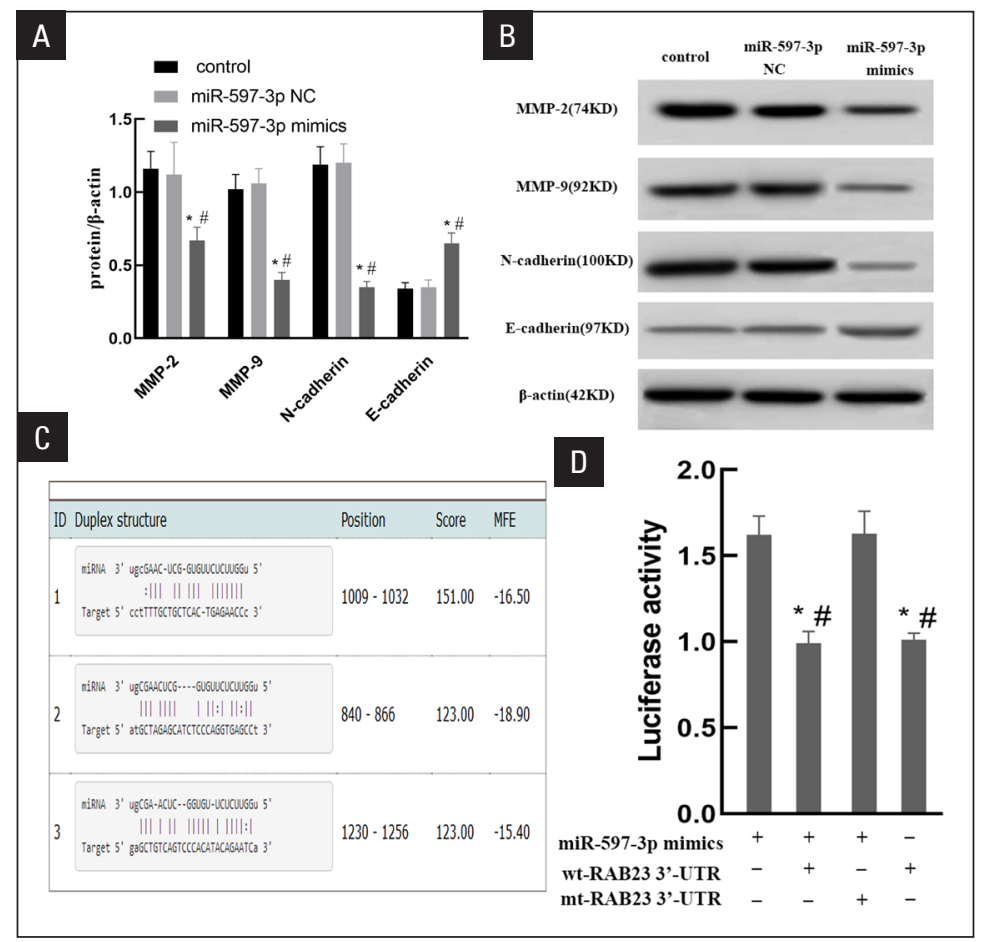

Figure 3. A, B. The level of MMP-2, MMP-9, N-cadherin, and E-cadherin protein in the invasion and migration. Data are expressed as mean $\pm S D(n=3)$. ${ }^{*} p<0.05$ vs. control group; ${ }^{*} p<0.05$ vs. miR-597-3p NC group. C. The suspected binding of miR-597-3p with the wild type (WT) 3'UTR region of RAB23 mRNA was shown by the miRTarase Database (www.targetscan.org) ( $n=3$ ). D. The luciferase activity after miR-597-3p mimics co-transfected with wt-RAB23 3'-UTR or mt-RAB23 3'-UTR. Data are expressed as mean $\pm S D(n=3)$. ${ }^{*} p<0.05$ vs. transfected with miR-597-3p mimics; ${ }^{*} p<0.05$ vs. transfected with miR-597-3p mimics co-transfected with mt-RAB23 3'-UTR 
used. The miRTarase Database predicted that the RAB23 3'-UTR region had three binding points of miR-597-3p (Fig. 3C). The Luciferase reporter showed that, notably, the luciferase activity of SW579 cells transfected with wt RAB23 3'-UTR and miR-597-3p mimics was lower than transfected with miR-597-3p mimics only $(p<0.05$, Fig. 3D). However, luciferase activity of SW579 cells showed no significant variation after transfection with mt RAB23 3'-UTR ( $p>0.05$, Fig. 3D).

\section{Discussion}

Thyroid carcinoma is a common endocrine tumour. Along with the development of medical conditions, the therapeutic methods for TC patients have improved in most regions of the word, but TC morbidity and mortality has been continuously increasing in recent decades $[15,16]$. It has been recently indicated that a relationship exists between miRNA and TC; the abnormal expression of these miRNAs are conducive to the biological changes in TC cells [17]. However, the ideal genetic marker for TC testing has not yet been discovered, and the roles of miR-597-3p in TC remain unclear. Therefore, in the current study, we found that overexpression of miR-597-3p could down-regulate the expression of RAB23, and it decreased the abilities of SW579 cells in terms of proliferation, invasion, and migration.

MiRNAs perform a significant function in various biological processes of tumour cells by targeting the 3 -untranslated region and regulating the translation of their target miRNAs [18]. Several researchers have shown that miRNAs can regulate the expression of a variety of genes involved in tumour progression [19]. More and more evidence has shown that miRNAs can serve as either tumour suppressors or oncogenes in biological processes such as proliferation, invasion, and migration of tumour cells [20]. Wang et al. [21] indicated that miR-338-3p is implicated in prostate cancer and that forced expression of miR338-3p suppressed tumour growth in vivo as well as proliferation, migration, and invasion in vitro. Subsequently, it was shown that miR338-3p could bind directly to the 3 '-untranslated region of RAB23 mRNA and down-regulate the expression of RAB23. In addition, Melissa et al. [22] discovered that miR-466 was under-expressed in PCa tissue compared to normal tissues, and miR-466 suppressed PCa metastasis and growth by directly targeting bone-related transcription factor RUNX2. Our study showed that the expression of miR-597-3p in SW579 cells was significantly increased compared with the control group and miR-597-3p NC group by 1.45 fold and 1.44 fold after transfection with miR-597-3p mimics, suggesting that the miR-597-3p mimics were success- fully transfected in SW579 cells and up-regulated miR-597-3p expression.

The SW579 cell viabilities were measured through the CCK-8 assay, and the result showed that the SW579 cell viabilities sharply decreased and was lower than in the miR-597-3p NC group $(92.12 \%)$ and control group $(90.28 \%)$ after transfection with miR-597-3p mimics, indicating that up-regulation of the expression miR-597-3p could suppress the viabilities of SW579 cells. In addition, the Transwell chamber assay was used to evaluate the abilities of invasion and migration of cancer cells; the results indicated that the invasion and migration abilities of SW579 cells were significantly decreased compared with the control group and miR-597-3p NC group after transfection with miR-597-3p mimics, indicating that miR-597-3p could regulate the invasion and migration abilities of SW579 cells and be involved in the progress of TC. Some researchers also illuminated the role of miRNAs in TC. The expression of miR-21 was up-regulated in papillary thyroid carcinoma cells and tissues, and then the expression of miR-21 was down regulated by PI3K/Akt pathway and invasion mediated epithelial-mesenchymal transition (EMT), thus inhibiting the proliferation of papillary thyroid carcinoma cell [23].The above result different with ours study, the different miRNAs may play distinctive role in thyroid carcinoma, whereafter, the report found that the miR-1280 levels were significantly lower in TC cells and tissues compare with healthy thyroid cells and adjacent normal tissues, whereas overexpression of miR-1280 inhibited cells proliferation and invasion of TC cells, besides, overexpression of miR-1280 inhibited ERK and ER $\alpha$ pathway of TC cells [24]. In order to deeply explore the effect of the invasion and migration of SW579 cells after transfected with miR-597-3p minics, the proteins levels of correlated with invasion and migration. The MMPs family is usually found to take part in the degradation of extracellular matrix, which is involved in cell invasion and migration. MMP-2 and MMP-9 are the two most generally researched members of this family. Further study indicated that TRIB1-mediated invasion and migration of colorectal cancer cells requires up-regulation of the expression of MMP-2 by activating the ERK and FAK/Src pathway [25]. EGCG and IIF are also the most effective treatments in reducing the expression of marks of migration and invasion such as MMP-2, MMP-9, and CD44 in MCF-7, MCF-7 TAM, and MDA-MB-231 cells [26]. N-cadherin could promote the EMT and angiogenesis of tumours, and E-cadherin could promote tight junction intercellular and inhibit migration of tumour cells. All of these play a key role in EMT and are significant in tumour cell invasion and migration in various tumour types. The research revealed that procollagen-lysine, 2-oxoglutar- 
ate 5-dioxygenase 2 (PLOD2) activated the PI3K/AKT signalling pathway and up-regulated the expression of EMT regulators such as N-cadherin and vimentin and down-regulated the expression of E-cadherin [27]. Feng et al. [28] found that down-regulated expression of $\mathrm{N}$-cadherin, NF- $\kappa \mathrm{B}$ p 65 , and vimentin and up-regulation of E-cadherin decreased the abilities of invasion and migration in lung cancer A549 cells. Our study has also shown that the overexpression of miR-597-3p decreased the abilities of invasion and migration in SW579 cells, indicating that up-regulated expression of miR-597-3p would decrease the expression of invasion and migration factors and negatively regulate EMT transcription.

In recent years, the role of RAB23 in malignancy has attracted keen interest among researchers. The latest reports reveal that RAB23 is significantly up-regulated in gastric cell lines, and miR-802 could directly target RAB23. Additionally, overexpression of miR-802 suppresses the invasion and migration of gastric cell lines [29]. In oesophageal squamous cell carcinoma cells the expression of RAB23 is up-regulated, and RAB23 knockdown could inhibit the growth, migration, and invasion, suggesting that RAB23 could be a therapeutic target of oesophageal squamous cell carcinoma [30]. Meanwhile, in hepatocellular carcinoma cells, miR-429 reversed the EMT to MET and decreased the migratory capacity through targeting of RAB23 [31]. In the present study, we found that the expression of RAB23 was up-regulated in SW579 cells after transfected with miR-597-3p mimics; subsequently, Luciferase reporter revealed that the RAB23 was the target of miR-597-3p in SW579 cells, suggesting that miR-597-3p might target-regulate the expression of RAB23 and decrease the abilities of proliferation, invasion, and migration in SW579 cells.

\section{Conclusion}

Our study demonstrated that overexpression of miR-597-3p could target-regulate the expression of RAB23, and might, through that mechanism, decrease the expression of MMP-2, MMP-9, and N-cadherin and increase the expression of E-cadherin, and then suppress the abilities of proliferation, invasion, and migration in SW579 cells, suggesting that miR-597-3p might become a target gen to cure TC.

\section{Funding}

This study was supported by the Affiliated Hospital of North Sichuan Medical College — grant No. 2020ZD015.

\section{References}

1. Liu K, Huang W, Yan DQ, et al. Overexpression of long intergenic noncoding RNA LINC00312 inhibits the invasion and migration of thyroid cancer cells by down-regulating microRNA-197-3p. Biosci Rep. 2017; 37(4), doi: 10.1042/BSR20170109, indexed in Pubmed: 28539331.
2 Pishkari S, Paryan M, Hashemi M, et al. The role of microRNAs in different types of thyroid carcinoma: a comprehensive analysis to find new miRNA supplementary therapies. J Endocrinol Invest. 2018; 41(3): 269-283, doi: 10.1007/s40618-017-0735-6, indexed in Pubmed: 28762013.

3. Sanabria A, Kowalski LP, Shah JP, et al. Growing incidence of thyroid carcinoma in recent years: Factors underlying overdiagnosis. Head Neck. 2018; 40(4): 855-866, doi: 10.1002/hed.25029, indexed in Pubmed: 29206325

4. Lin $\mathrm{Y}, \mathrm{Ma} \mathrm{Q}$, Li L, et al. The CXCL12-CXCR4 axis promotes migration, invasiveness, and EMT in human papillary thyroid carcinoma B-CPAP cells via NF- $\kappa$ B signaling. Biochem Cell Biol. 2018; 96(5): 619-626, doi: 10.1139/bcb-2017-0074, indexed in Pubmed: 29316404.

5. Au JK, Alonso J, Kuan EC, et al. Primary Squamous Cell Carcinoma of the Thyroid: A Population-Based Analysis. Otolaryngol Head Neck Surg. 2017; 157(1): 25-29, doi: 10.1177/0194599817698436, indexed in Pubmed: 28397584.

6. Natalia MA, Alejandro GT, Virginia TVI, et al. MARK1 is a Novel Targe for miR-125a-5p: Implications for Cell Migration in Cervical Tumor Cells. Microrna. 2018; 7(1): 54-61, doi: 10.2174/221153660666617102416 0244, indexed in Pubmed: 29076440.

7. Li D, Zhang Y, Zhang He, et al. CADM2, as a new target of miR-10b, promotes tumor metastasis through FAK/AKT pathway in hepatocellular carcinoma. J Exp Clin Cancer Res. 2018; 37(1): 46 doi: 10.1186/s13046-018-0699-1, indexed in Pubmed: 29506532.

8. Zheng S, Zhang X, Wang X, et al. Downregulation of miR-138 predicts poor prognosis in patients with esophageal squamous cell carcinoma. Cancer Biomark. 2017; 20(1): 49-54, doi: 10.3233/CBM-170079, indexed in Pubmed: 28759955.

9. Xue KC, Hu DD, Zhao L, et al. MiR-577 inhibits papillary thyroid carcinoma cell proliferation, migration and invasion by targeting SphK2. Eur Rev Med Pharmacol Sci. 2017; 21(17): 3794-3800, indexed in Pubmed: 28975989

10. Wang WW, Chen B, Lei CB, et al. miR-582-5p inhibits invasion and migration of salivary adenoid cystic carcinoma cells by targeting FOXC1. Jpn J Clin Oncol. 2017; 47(8): 690-698, doi: 10.1093/jjco/hyx073, indexed in Pubmed: 28591777.

11. Li Z, Hu Y, Zeng Q, et al. Circular RNA MYLK promotes hepatocellular carcinoma progression by increasing Rab23 expression by sponging miR-362-3p. Cancer Cell Int. 2019; 19: 211, doi: 10.1186/s12935-019-0926-7, indexed in Pubmed: 31413665.

12. Hor CHH, Tang BL, Goh ELK. Rab23 and developmental disorders. Rev Neurosci. 2018; 29(8): 849-860, doi: 10.1515/revneuro-2017-0110, indexed in Pubmed: 29727300.

13. Wang $\mathrm{Y}$, Oin $\mathrm{H}$. miR-338-3p targets and suppresses tumorigenicity of prostate cancer cells. Am J Cancer Res. 2018; 8(12): 2564-2574, indexed in Pubmed: 30662812

14. Zhang W, Yu F, Wang Yu, et al. Rab23 promotes the cisplatin resistance of ovarian cancer via the Shh-Gli-ABCG2 signaling pathway. Oncol Lett. 2018; 15(4): 5155-5160, doi: 10.3892/ol.2018.7949, indexed in Pubmed: 29552151.

15. Wang CJ, Yang D, Luo YW. Recombinant ING4 suppresses the migration of SW579 thyroid cancer cells via epithelial to mesenchymal transition. Exp Ther Med. 2015; 10(2): 603-607, doi: 10.3892/etm.2015.2515, indexed in Pubmed: 26622361

16. Kalra R, Pawar R, Hasija S, et al. Frontal bone metastasis from an occult follicular thyroid carcinoma: Diagnosed by FNAC. J Cytol. 2017; 34(1): 59-61, doi: 10.4103/0970-9371.197623, indexed in Pubmed: 28182063.

17. Yuan Q, Liu Y, Fan Y, et al. LncRNA HOTTIP promotes papillary thyroid carcinoma cell proliferation, invasion and migration by regulating miR-637. Int J Biochem Cell Biol. 2018; 98: 1-9, doi: 10.1016/j. biocel.2018.02.013, indexed in Pubmed: 29474928

18. van Beijnum JR, Giovannetti E, Poel D, et al. miRNAs: micro-managers of anticancer combination therapies. Angiogenesis. 2017; 20(2): 269-285, doi: 10.1007/s10456-017-9545-x, indexed in Pubmed: 28474282

19. Dong F, Xu T, Shen Y, et al. Dysregulation of miRNAs in bladder cancer: altered expression with aberrant biogenesis procedure. Oncotarget. 2017; 8(16): 27547-27568, doi: 10.18632/oncotarget.15173, indexed in Pubmed: 28187437.

20. Adhami M, Haghdoost AA, Sadeghi B, et al. Candidate miRNAs in human breast cancer biomarkers: a systematic review. Breast Cancer. 2018; 25(2): 198-205, doi: 10.1007/s12282-017-0814-8, indexed in Pubmed: 29101635.

21. Wang $Y$, Qin H. miR-338-3p targets and suppresses tumorigenicity of prostate cancer cells. Am J Cancer Res. 2018; 8(12): 2564-2574, indexed in Pubmed: 30662812

22. Colden M, Dar AA, Saini S, et al. MicroRNA-466 inhibits tumor growth and bone metastasis in prostate cancer by direct regulation of osteogenic transcription factor RUNX2. Cell Death Dis. 2017; 8(1): e2572, doi: 10.1038/cddis.2017.15, indexed in Pubmed: 28125091.

23. Zang C, Sun J, Liu W, et al. miRNA-21 promotes cell proliferation and invasion via VHL/PI3K/AKT in papillary thyroid carcinoma. Hum Cell. 2019; 32(4): 428-436, doi: 10.1007/s13577-019-00254-4, indexed in Pubmed: 31161410 
24. Meng D, Li Z, Ma X, et al. MicroRNA-1280 modulates cell growth and invasion of thyroid carcinoma through targeting estrogen receptor $\alpha$. Cell Mol Biol (Noisy-le-grand). Cell Mol Biol (Noisy-le-grand). 2016; 62(3): 1-6, indexed in Pubmed: 27064865.

25. Wang Y, Wu N, Pang Bo, et al. TRIB1 promotes colorectal cancer cell migration and invasion through activation MMP-2 via FAK/Src and ERK pathways. Oncotarget. 2017; 8(29): 47931-47942, doi: 10.18632/oncotarget.18201, indexed in Pubmed: 28624785.

26. Farabegoli F, Govoni M, Spisni E, et al. EGFR inhibition by (-)-epigallocatechin-3-gallate and IIF treatments reduces breast cancer cell invasion. Biosci Rep. 2017; 37(3), doi: 10.1042/BSR20170168, indexed in Pubmed: 28465354.

27. Song Ye, Zheng S, Wang J, et al. Hypoxia-induced PLOD2 promotes proliferation, migration and invasion via PI3K/Akt signaling in glioma. Oncotarget. 2017; 8(26): 41947-41962, doi: 10.18632/oncotarget.16710, indexed in Pubmed: 28410212.
28. Feng H, Lu JJ, Wang Y, et al. Osthole inhibited TGF $\beta$-induced epithelial-mesenchymal transition (EMT) by suppressing NF- $\kappa$ B mediated Snail activation in lung cancer A549 cells. Cell Adh Migr. 2017; 11(5-6): 464-475, doi: 10.1080/19336918.2016.1259058, indexed in Pubmed: 28146373.

29. Zhang XY, Mu JH, Liu LY, et al. Upregulation of miR-802 suppresses gastric cancer oncogenicity via targeting RAB23 expression. Eur Rev Med Pharmacol Sci. 2017; 21(18): 4071-4078, indexed in Pubmed: 29028094.

30. Cheng L, Yang F, Zhou B, et al. RAB23, regulated by miR-92b, promotes the progression of esophageal squamous cell carcinoma. Gene. 2016; 595(1): 31-38, doi: 10.1016/j.gene.2016.09.028, indexed in Pubmed: 27659550.

31. Xue H, Tian GY. MiR-429 regulates the metastasis and EMT of HCC cells through targeting RAB23. Arch Biochem Biophys. 2018; 637: 48-55, doi: 10.1016/j.abb.2017.11.011, indexed in Pubmed: 29191386. 